\title{
Effective coverage measurement in maternal, newborn, child, and adolescent health and nutrition: progress, future prospects, and implications for quality health systems
}

\author{
Andrew D Marsh, Moise Muzigaba, Theresa Diaz, Jennifer Requejo, Debra Jackson, Doris Chou, Jenny A Cresswell, Regina Guthold, Allisyn C Moran, \\ Kathleen L Strong, Anshu Banerjee, Agnès Soucat, on behalf of the Effective Coverage Think Tank Group*
}

\begin{abstract}
Intervention coverage- the proportion of the population with a health-care need who receive care-does not account for intervention quality and potentially overestimates health benefits of services provided to populations. Effective coverage introduces the dimension of quality of care to the measurement of intervention coverage. Many definitions and methodological approaches to measuring effective coverage have been developed, resulting in confusion over definition, calculation, interpretation, and monitoring of these measures. To develop a consensus on the definition and measurement of effective coverage for maternal, newborn, child, and adolescent health and nutrition (MNCAHN), WHO and UNICEF convened a group of experts, the Effective Coverage Think Tank Group, to make recommendations for standardising the definition of effective coverage, measurement approaches for effective coverage, indicators of effective coverage in MNCAHN, and to develop future effective coverage research priorities. Via a series of consultations, the group recommended that effective coverage be defined as the proportion of a population in need of a service that resulted in a positive health outcome from the service. The proposed effective coverage measures and care cascade steps can be applied to further develop effective coverage measures across a broad range of MNCAHN services. Furthermore, advances in measurement of effective coverage could improve monitoring efforts towards the achievement of universal health coverage.
\end{abstract}

\section{Introduction}

Universal health coverage is at the centre of Sustainable Development Goal (SDG) 3: "to ensure healthy lives and promote wellbeing for all, at all ages", and is described as the key driver for achieving health-related targets. ${ }^{1}$

Monitoring progress towards achieving universal health coverage requires metrics that capture information on the proportion of the population in need of care that receives health services at a sufficient level of quality to yield the intended health benefits. Effective coverage adds the dimension of quality of care to the measurement of intervention coverage and aims to better capture the potential health benefits of an intervention. ${ }^{2}$ However, despite the potential of the effective coverage approach to overcome some of the limitations of intervention coverage, consensus has not been reached on its definition, methodological approaches for measurement, and how indicators of effective coverage should be interpreted to inform global monitoring and national programmes and policies. This variability in definition leads to confusion as to what actions should be taken to monitor and improve quality of care. Due to varying approaches to estimate effective coverage, the data used to measure effective coverage vary greatly from selfreported population surveys, surveys with biomarkers, observations, facility surveys, and routine data collected through the Health Management Information Systems. Although the data used to estimate effective coverage often depend on the concept being measured (eg, content of visit, readiness of health-care facility to provide care), sometimes the same concepts use different data sources.
Standardisation of methods to measure effective coverage is urgently needed to ensure that health programmers and policy makers can take informed actions to improve quality of care for maternal, neonatal, child, and adolescent health and nutrition (MNCAHN).

In 2019, WHO and UNICEF convened a group of experts, the Effective Coverage Think Tank Group, to establish standardised definitions and measurement approaches for effective coverage, initiate discussions on effective coverage indicators for MNCAHN, and to develop priorities for future research on effective coverage. The Effective Coverage Think Tank series included four video teleconferences, between March and April, 2019, and an in-person meeting in May, 2019. Participants included 98 experts in the fields of qualityof-care measurement, monitoring and evaluation, epidemiology, and research. They were selected to be both geographically diverse and include content expertise across the range of MNCAHN components. Participant inputs were solicited in advance of each video teleconference through a combination of group email correspondence and online survey tools. Participation was moderated through online video conferencing, with a record of each call saved at its completion. The in-person meeting was hosted by WHO and UNICEF and included a representative group of experts selected according to the same criteria described above. The purpose of the meeting was to synthesise the experience from the video teleconferences and make recommendations for defining and measuring effective coverage. This Health Policy paper summarises the
Lancet Glob Health 2020; 8: e730-36 *Listed at the end of the Health Policy

Baltimore, MD, USA (A D Marsh PhD); Department of Maternal, Newborn, Child and Adolescent Health and Ageing (M Muzigaba PhD, T Diaz MD, R Guthold PhD, A C Moran PhD, K L Strong PhD, A Banerjee PhD), Department of Health Systems Governance and Financing (A Soucat PhD), and Department of Sexual and Reproductive Health and Research including the UNDPUNFPA-UNICEF-WHO-World Bank Special Programme of Research, Development and Research Training in Human Reproduction (D Chou MD, J A Cresswell PhD), World Health Organization, Geneva, Switzerland; Division of Data, Analysis, Planning and Monitoring (J Requejo PhD) and Health Division (Prof D Jackson DSc), United Nations Children's Fund, Headquarters, New York, NY, USA; and School of Public Health, University of the Western Cape, Cape Town, South Africa (Prof D Jackson) Correspondence to: DrTheresa Diaz, Epidemiology Monitoring and Evaluation Unit, Department of Maternal Newborn, Child and Adolescent Health and Ageing, World Health Organization, Geneva 1202, Switzerland tdiaz@who.int 
Panel 1: Examples of effective coverage definitions and measurement approaches

Effective coverage has been variously defined or measured:

- As the fraction of possible health gain an individual with a health-care need can expect to receive from the health system, formalised as a combination of need, use, and quality ${ }^{2}$

- By adjusting intervention coverage levels according to service readiness, ${ }^{5}$ quality of care provided, ${ }^{6}$ or health outcomes achieved ${ }^{7}$

- As a single indicator of overall health-system performance, calculated as a composite measurement of coverage across a range of interventions ${ }^{8}$

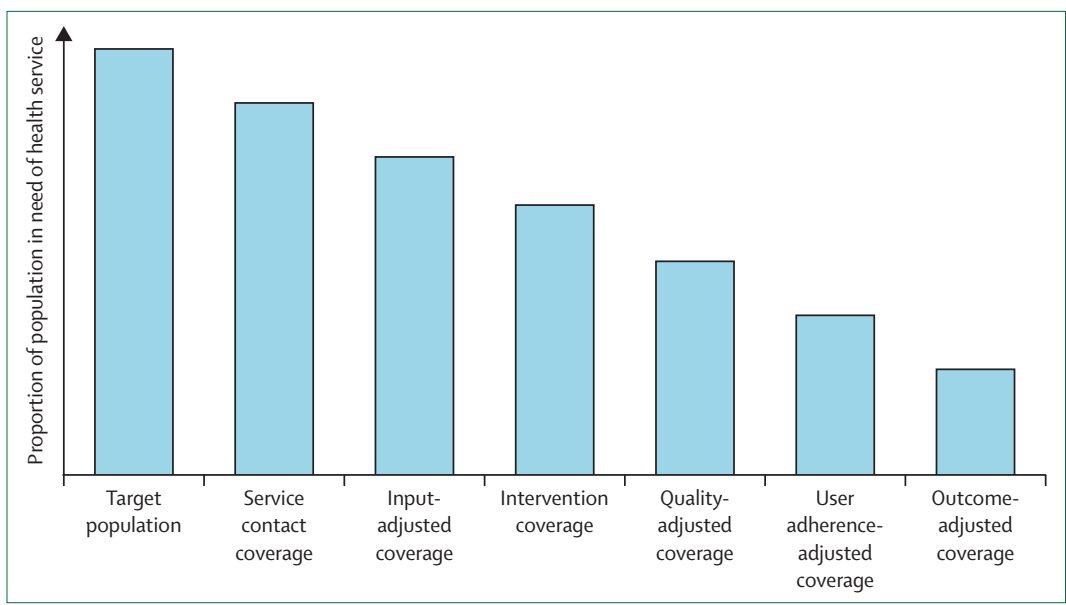

Figure 1: Proposed standardised cascade for measuring effective coverage Adapted from Amouzou and colleagues. ${ }^{4}$

discussion and recommendations from the Effective Coverage Think Tank Group.

\section{Findings \\ Evidence synthesis}

We examined scoping and systematic reviews on effective coverage frameworks, including a recent scoping review of effective coverage applications ${ }^{3}$ and systematic review of effective coverage in MNCHN. ${ }^{4}$ In the scoping review, Jannati and colleagues ${ }^{3}$ searched seven databases for publications on effective coverage applications for assessment of health-system performance published before May, 2017, with no start date restriction, and identified 18 studies, four of which examined effective coverage of the health system as a whole and 14 assessed effective coverage for specific interventions. In their systematic review, Amouzou and colleagues $^{4}$ restricted their focus to applications of effective coverage in MNCAHN and reproductive health. They identified 36 studies published between January, 2000, and October, 2017, 30 of which were not included in Jannati and colleagues' scoping review, and highlighted the considerable variability between studies relating to data sources for effective coverage, indicator definitions, and analytical approaches. We shared our findings (panel 1) with Effective Coverage
Think Tank experts, providing a common background for discussions.

\section{Recommendations for health-service coverage cascades and terminology}

Participants recommended that effective coverage be explained using health-service coverage cascades applied at the population level. Cascades provide a tool for assessing health-system performance across the sequence of interactions between patients and the health system. ${ }^{9}$ The generic cascade proposed by Amouzou and colleagues ${ }^{4}$ which builds on the Tanahashi framework ${ }^{10}$ for evaluating health-service coverage and allows for population-level assessment of health services along the MNCAHN continuum of care, was supported by Effective Coverage Think Tank participants for its flexibility to adapt across a range of health services.

The Think Tank Group proposed the following adaptation of Amouzou and colleagues' cascade steps (figure 1) and their definitions, with illustrative examples for MNCAHN interventions. Step 1 is the target population: identifying the population with a specific health need. Step 2 is service contact coverage: the proportion of the population in need who come into contact with the (relevant) health service. Step 3 is inputadjusted coverage: the proportion of the population in need who come into contact with a health service that is ready to provide care. Readiness requires that all inputs necessary to provide the service are available in sufficient quantity and quality and are usable at the time of the visit. The specific components will vary with the service and the context in which it is provided (eg, health facility, community, school). Step 4 is intervention coverage: the proportion of the population in need who come into contact with a service that is ready and that receives the service. Step 5 is quality-adjusted coverage: the proportion of the population in need who come into contact with a service that is ready and that receives the service according to quality-of-care standards. Quality-of-care standards constitute what is expected to be delivered to maximise the potential positive health outcome and are commonly measured by assessing the content of the health service relative to guidelines (eg, WHO guidelines). ${ }^{11-14}$ Meeting these standards implies that the necessary practices have been followed while unnecessary or harmful practices have been avoided (eg, unnecessary antibiotic use) Furthermore, quality of care extends beyond adherence to guidelines to also consider whether the service was provided respectfully. ${ }^{15}$ Step 6 is user adherence-adjusted coverage: the proportion of the population in need who receives the service according to quality-of-care standards and that adheres to provider instructions. For newborn babies and young children, this adherence includes caregiver adherence to provider instructions. This step might not apply to services that require no additional user action after they have been delivered (eg, vaccination). Finally, step 7 is outcome-adjusted coverage: the 
proportion of the population in need who receives the service according to quality-of-care standards, adheres to provider instructions, and has the expected health outcome. For curative interventions, a positive health outcome is assessed by a return to good health, while a positive health outcome for preventive and promotive services is the absence of health loss.

\section{Recommendations for MNCAHN effective coverage definitions and measures}

Recommendations from the Think Tank participants for the definition and measurement of effective coverage are shown in panel 2. Several considerations are important when selecting effective coverage measures and developing cascades. First, measuring effective coverage through quality-adjusted or outcome-adjusted coverage without reporting the additional steps of the associated cascade might be best suited for global and national monitoring because it provides an overall impression of effective coverage in a country. Second, the full cascade is most relevant for monitoring at subnational and facility levels, which might need detailed information to identify bottlenecks in service provision for determining appropriate remedial actions. ${ }^{16}$ Third, the level at which data are analysed should also be consistent with their intended use. For example, districtlevel cascades and their assessments could appropriately inform decision making at the district level but might not always be suitable for making targeted changes at each individual facility within the district. Finally, a distinction should be made between what can be currently measured given data availability and techniques for linking data sources, and what could ideally be measured in the future if investments are made in the effective coverage research agenda and in-country health information systems.

\section{Potential data sources proposed for effective coverage measures}

Wherever possible, effective coverage measures should rely on validated data. A combination of data sources might be necessary to calculate indicators (eg, health facility assessments, household surveys, and routine Health Management Information System and administrative data). For example, a household survey might collect the epidemiological data necessary to quantify the target population (eg, incidence, prevalence) while also collecting data on care-seeking behaviours for estimating service contact and receipt of services necessary for estimating intervention coverage. These data could be combined with health facility assessment results to estimate input-adjusted coverage and potentially qualityadjusted coverage if the health facility assessment included direct observation of services. Although data on user adherence are generally more challenging to obtain, such data might be estimated according to reported data from similar settings. Finally, outcome data might be
Panel 2: Recommendations from the Effective Coverage Think Tank Group for definition and measures of effective coverage for maternal, newborn, child, and adolescent health and nutrition

- Effective coverage is defined as the proportion of a population in need of a service that had a positive health outcome from the service

- Effective coverage is ideally measured as outcome-adjusted coverage in the healthservice coverage cascade; this type of measure is potentially feasible to produce when a target population needs a specific health service with proven effectiveness and for which the health impact can be directly linked to the specific service-eg, children living with HIV requiring antiretroviral therapy and with viral load suppression

- For routine preventive or promotive health services, such as counselling services, or antenatal and postnatal care visits during which multiple interventions are delivered, each of which might be linked to the same or different health outcomes, outcomeadjusted coverage is not an ideal effective coverage measure; a more amenable measure for these health services is quality-adjusted coverage, which might be a proxy (indirect) measurement of effective coverage; quality-adjusted coverage measures can capture information on the timeliness, content, and quality of the health service provided based on guidelines and recommendations

- Quality-adjusted coverage might also serve as a proxy for effective coverage in cases where outcome-adjusted coverage could potentially be measured but the added value of these data is outweighed by the resources necessary to obtain them (eg, immune response resulting from vaccination that is complicated by challenges attributing such a response to vaccination rather than pathogen exposure)

- Mortality measures are generally not a substitute for measuring effective coverage for several reasons-eg, mortality often results from a combination of factors, many of which are outside the health system and aggregated mortality cannot be used to identify where the health system has failed; we also recognise that substantial data gaps exist for cause-specific mortality data (eg, case fatality rates)

- Under a few circumstances, mortality measures might be used to measure effective coverage when the cause of death is directly attributable to (the absence of) a specific health service-eg, the incidence of intrapartum stillbirth (occurring during the labour and childbirth period) has been used as a measure of effective coverage of intrapartum care; also, when the incidence and mortality of lethal conditions with known treatments are tracked (eg, some cancers are monitored through cancer registries), use of mortality-to-incidence ratios might be possible (eg, to assess the health impact or benefit of cancer screening and treatment programmes)

Whether effective coverage is measured directly through outcome-adjusted coverage or indirectly through quality-adjusted coverage as a proxy, effective coverage is represented by a single point along the cascade rather than as the entire cascade itself

collected through disease surveillance or populationbased surveys. These illustrative data sources provide an example of how cascade steps might be assessed. However, selecting ideal data sources depends on the service for which effective coverage is being assessed. Additional considerations regarding potential data sources for effective coverage measures have been published elsewhere. ${ }^{3,4,17,18}$

\section{Applying effective coverage and health-service coverage cascades to MNCAHN}

The Effective Coverage Think Tank Group developed several example cascades for MNCAHN to show how effective coverage should be applied. 


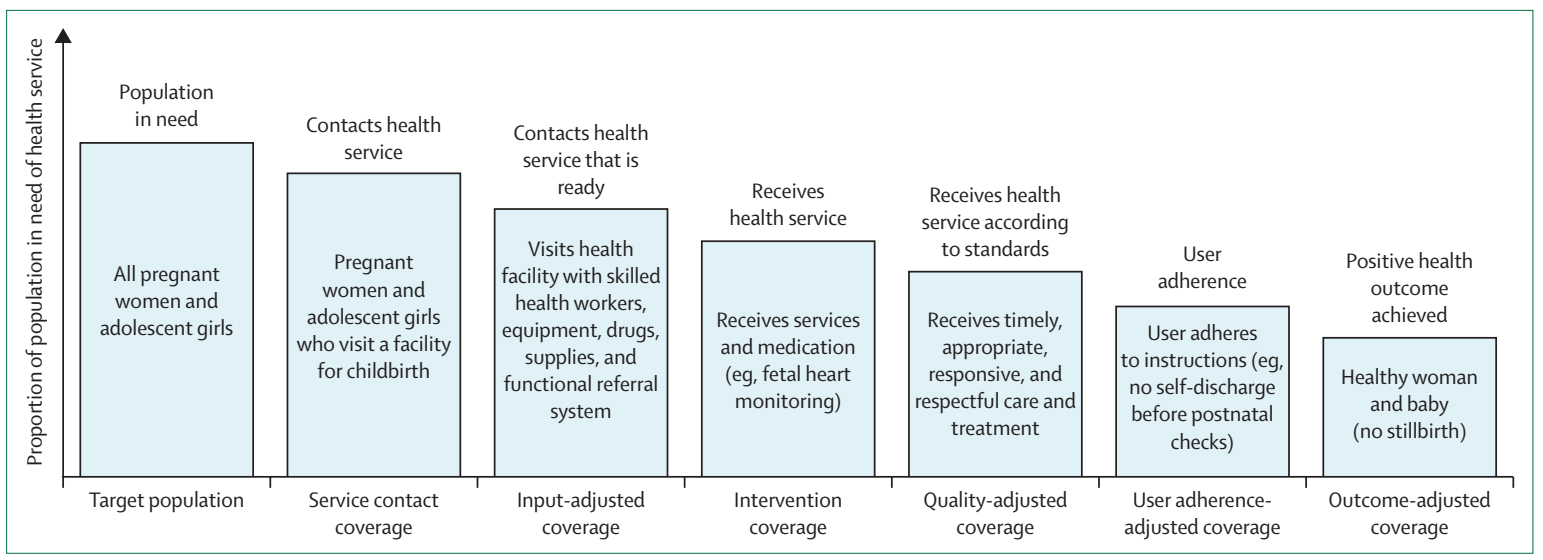

Figure 2: Health-service coverage cascade for routine childbirth care

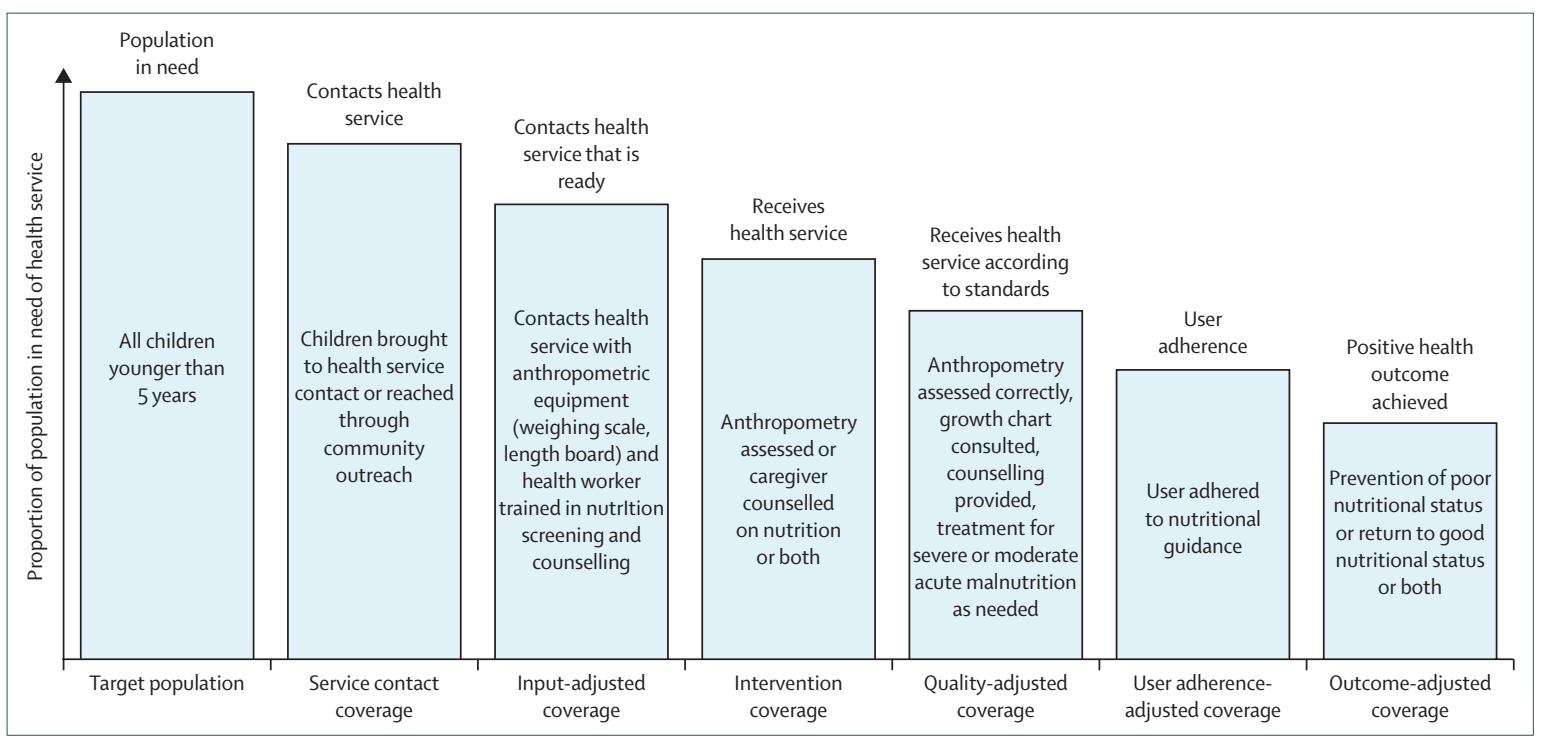

Figure 3: Health-service coverage cascade for growth monitoring, promotion, and treatment of malnutrition in children

\section{Example in maternal and newborn health}

For routine antenatal, childbirth, and postnatal care, quality-adjusted coverage is the preferred measure of effective coverage (figure 2) because of the challenge of attributing maternal and neonatal mortality across these services. However, for complications, outcome-adjusted effective coverage would be an appropriate measure. Because measurement of all complications is not practical, a tracer complication can be selected-eg, for post-partum haemorrhage, the outcome-adjusted coverage measure of controlled post-partum bleeding could be used.

\section{Example in child health}

The discussion of effective coverage for child health included interventions for both well children, including children with disabilities (prevention of disease, promotion of growth and development), and sick or injured children (treatment of acute and chronic diseases and injuries).$^{19}$ Each of these domains includes a suite of interventions. A separate cascade and measurement of effective coverage could be developed for each intervention; however, resource limitations in different contexts would make tracking of effective coverage of all interventions simultaneously impractical. When multiple interventions are delivered through the same platform (eg, Integrated Management of Childhood Illness ${ }^{20}$ or Integrated Community Case Management ${ }^{21}$ ), selection of one intervention as a tracer for the overall contact might be appropriate.

Quality-adjusted coverage was the preferred effective coverage measure for care of a sick child (acute or chronic illness), prevention of disease, and promotion of growth and development. This choice reflects the challenge of attributing contributions of several interventions to a single health outcome of child survival or wellbeing. Additionally, given the strong association between delivering some high-quality interventions and subsequent positive health outcome (eg, immunisations and 


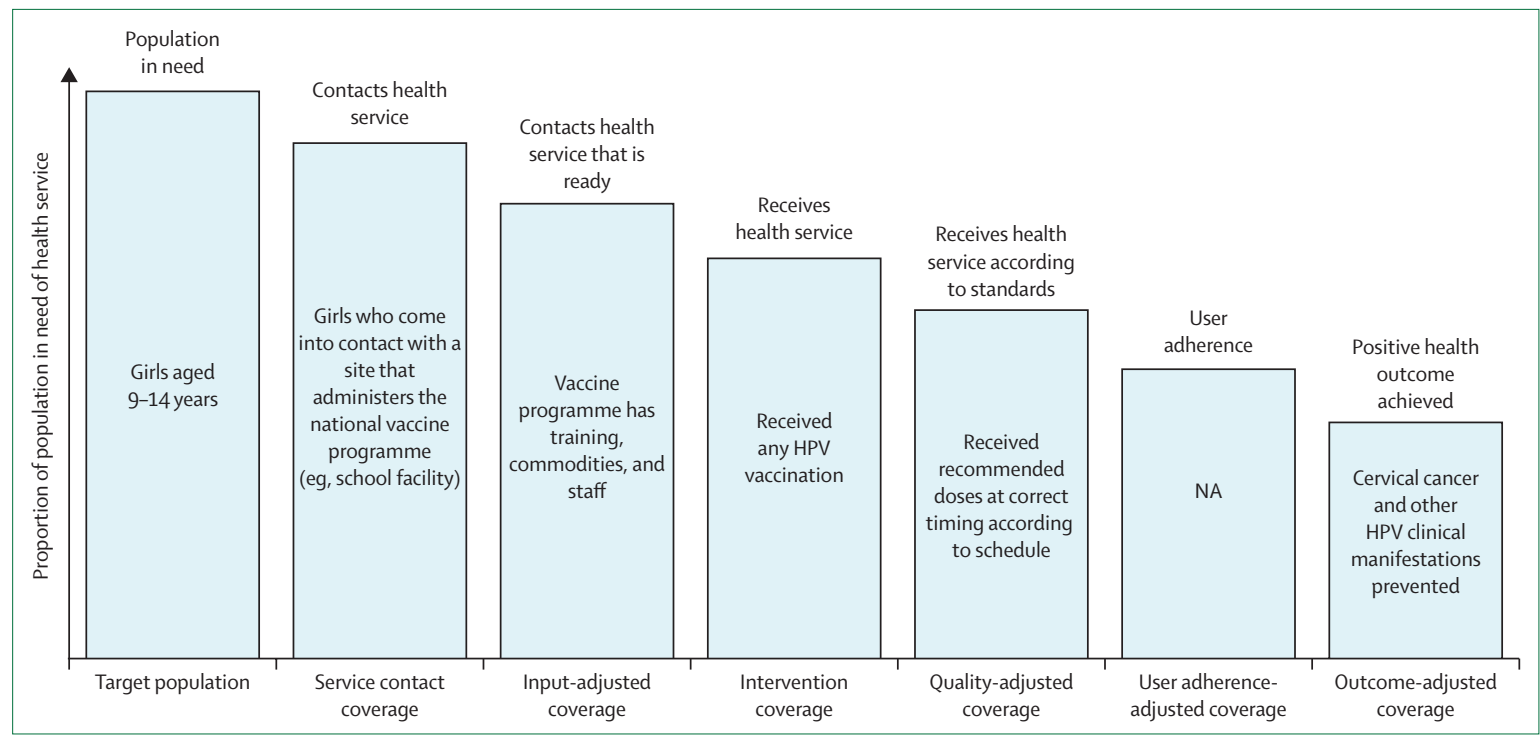

Figure 4: Health-service coverage cascade for HPV immunisation in girls aged 9-14 years

$\mathrm{HPV}=$ human papillomavirus. $\mathrm{NA}=$ not applicable.

seroconversion), quality-adjusted coverage can be a suitable proxy for outcome-adjusted coverage.

A cascade for growth monitoring and treatment of malnutrition in children is shown in figure 3 . The cascade includes a measure of outcome-adjusted coverage, but given the issues discussed here, a qualityadjusted coverage measure could also be appropriate.

\section{Example in adolescent health}

The approach to measuring effective coverage for adolescent health differs from the previous two groups because many interventions in adolescent health are delivered outside of the formal health system or not on an individual basis. The Think Tank Group selected interventions from among those delivered either through the health or school-based programmes and identified them on the basis of the burden of disease. One intervention selected was vaccination of adolescent girls aged 9-14 years against human papillomavirus. A cascade for this intervention is shown in figure 4.

\section{Discussion}

The outcomes of the Effective Coverage Think Tank Group are a step towards improving effective coverage measurement and our ability to assess health outcomes of proven interventions. At the global level, these recommendations on effective coverage will inform efforts to improve the universal health coverage service coverage index-the official measure for SDG indicator 3.8.1, 22,23 which has been criticised for not including measures of effective coverage.

The proposed health-service coverage cascade has four important caveats. First, the cascade does not explicitly account for the range of underlying reasons for gaps that might occur between identifying the target population in the first step of the cascade and the second step that captures who sought or received needed care. These reasons can include a mixture of additional demand and supply factors. ${ }^{24-26}$ If this gap is large, it should trigger further investigation into causal factors. Second, adjustment for user adherence is challenging to measure. Poor adherence to prescribed treatment can result in poor outcomes; however, many factors outside the health system can affect adherence. Third, the cascade does not include user experience as its own component. User experience is a measure of quality of care, yet measuring it is difficult and prone to bias. ${ }^{27}$ Finally, the cascade assumes that each step must occur to have the maximum positive health outcome. Notable exceptions exist in which a positive health outcome might occur in the absence of any health service (eg, recovery from illness or malnutrition in the absence of any treatment) or when the health service does not meet quality-of-care standards (eg, a healthy woman and neonate after a childbirth without a skilled birth attendant present).

The Effective Coverage Think Tank Group identified several research priorities. First, increased efficiency in the use of traditional data sources is required while also considering the potential for alternative data sources. Household surveys and health facility assessments provide valuable data; however, these instruments are administered only every 3-5 years, and have historically focused on women of reproductive age and children younger than 5 years for health-related issues. Further research is needed to determine how alternative data sources might effectively be used to complement these traditional data sources. These sources might include routinely collected administrative or Health Management Information System data, sentinel surveillance sites, or the growing body of data collected through innovative 
mobile health approaches. ${ }^{28}$ Because a single data source is unlikely to provide information on all steps in the cascade, improved coordination across data collection approaches and standardised methods and guidance for combining data from multiple sources are needed. ${ }^{29-31}$

Second, new approaches are required to improve the availability, validity, and reliability of data for measuring each step of the cascade. Examples include being able to identify specific target populations, such as preterm newborn babies requiring kangaroo mother care, because not all preterm newborn babies might be stable enough to initiate care. Capturing information on less tangible components of quality of care (eg, provider norms and attitudes) that affect demand issues, such as an individual's willingness to initially seek care and to stay in treatment if needed (step 2 of the cascade), is also a challenge.

Third, research is needed to understand the linkages between the steps in the cascade, which is especially important when measuring quality-adjusted coverage. National guidelines for service provision often include several activities that should be done during a visit, the monitoring of which might not be feasible in resourcelimited locations. Defining the subset of activities most linked with a health outcome will minimise the additional reporting burden while maintaining the usefulness of the measurement. Similarly, research is needed to understand and capture the resulting health effects when unnecessary or excessive practices are performed (eg, caesarean section without indication).

Fourth, implementation research is needed to ensure that the effective coverage frameworks proposed are responsive to the needs of decision makers and provide actionable information at the global, regional, national, and subnational levels and to determine what tools and capacity strengthening are needed in countries to collect, analyse, and use these data.

Finally, testing will help to further refine both effective coverage indicators and cascade steps, and to assess feasibility of measurement. As much as possible, these studies should assess inequalities in effective coverage by stratifying by key demographic variables. These stratified analyses should highlight the relevance of effective coverage in both low-income and high-income settings, where persistent inequities in health-service delivery remain.

\section{Contributors}

TD, AB, and AS conceived of the Effective Coverage Think Tank series. ADM, MM, TD, JR, DJ, DC, JAC, RG, ACM, and KLS organised and facilitated the discussions of the Effective Coverage Think Tank series. $\mathrm{ADM}$ and MM wrote the manuscript. All named authors and members of the Effective Coverage Think Tank Group reviewed the manuscript and approved the final draft.

The Effective Coverage Think Tank Group

Abdourahmane Diparidé Agbèrè, Ambrose Agweyu, Huda Alkitkat, Agbessi Amouzou, Fred Arnold, Peter Azzopardi, Mariame Gueye Ba Lenka Benova, Sk Masum Billah, Ann Blanc, Ties Boerma,

Cynthia Boschi-Pinto, Emily Carter, Liliana Carvajal-Aguirre,

Tamar Chitashvili, Siân Curtis, Inácio Crochemore M da Silva,
M Carolina Danovaro-Holliday, Bruce Dick, Shams El Arifeen, Khalifa Elmusharaf, Reina Engle-Stone, Mike English, Kate E Gilroy, Tanya Guenther, Ann Hagell, Lisa R Hirschhorn, Stephen Hodgins, Sayaka Horiuchi, Young Ae Jeong, Laura Kann, Jean-Louis Koulidiati, Margaret E Kruk, Rajesh Kumar, Etienne V Langlois, Joy E Lawn, Marzia Lazzerini, Hannah H Leslie, Tanya Marchant,

Catherine Mathews, Sanyukta Mathur, Claire-Helene Mershon, Diwakar Mohan, Ann-Beth Moller, Jean Pierre Monet, Alison Morgan, Melinda Munos, Holly Newby, Peter K Nguhiu, Ellen Piwoz, Thomas W Pullum, Pavani Ram, Rahul Rawat, Barbara Rawlins, Vahideh Sadeghi, Kuntal Saha, Joanna Schellenberg, Katherine E A Semrau, Florina Serbanescu, Kate Somers, Savitha Subramanian, Vandana Tripathi, Özge Tunçalp, Lara Vaz, Steve Wall, Wenjuan Wang, Ralf Weigel, William Weiss.

\section{Affiliations}

Centre Hospitalier Regional Lomé-Commune, Lomé, Togo (A D Agbèrè); Sylvanus Olympio Teaching Hospital, Lomé, Togo (A D Agbèrè); KEMRI-Wellcome Trust Research Programme, Nairobi, Kenya (A Agweyu, M English, P K Nguhiu); Portland State University, Portland, OR, USA (H Alkitkat); Johns Hopkins Bloomberg School of Public Health, Baltimore, MD, USA (A Amouzou, E Carter, D Mohan, M Munos, W Weiss); The Demographic and Health Surveys Program, ICF, Rockville, MD, USA (F Arnold, T W Pullum, W Wang); Maternal and Child Health Program, Burnet Institute, Melbourne, VIC, Australia (P Azzopardi); Wardliparingga Aboriginal Research Unit, South Australian Health and Medical Research Institute, Adelaide, SA, Australia (P Azzopardi); University of Melbourne, Melbourne, VIC, Australia (P Azzopardi, A Morgan); Faculty of Medicine, Pharmacy and Odontology, University Cheikh Anta Diop of Dakar-Sénégal, Dakar, Senegal (M G Ba); Gynecology and Obstetrics Clinic, University Teaching Hospital A Le Dantec, Dakar, Senegal (M G Ba); Institute for Tropical Medicine, Antwerp, Belgium (L Benova); International Centre for Diarrhoeal Disease Research, Dhaka, Bangladesh (S M Billah, S El Arifeen); The University of Sydney, Sydney, NSW, Australia (S M Billah); Population Council, New York, NY, USA (A Blanc, S Mathur); University of Manitoba, Winnipeg, MB, Canada (T Boerma); Universidade Federal Fluminense, Rio de Janeiro, Brazil

(C Boschi-Pinto); United Nations Children's Fund, New York, NY, USA (L Carvajal-Aguirre); University Research Co, Bethesda, MD, USA, (T Chitashvili); University of North Carolina, Chapel Hill, NC, USA (S Curtis); International Center for Equity in Health, Federal University of Pelotas, Pelotas, Brazil (I C M da Silva); World Health Organization, Geneva, Switzerland (M C Danovaro-Holliday, Y A Jeong, E V Langlois, A-B Moller, K Saha, Ö Tunçalp) Independent Consultant, Tannay, Switzerland (B Dick), Graduate Entry Medical School, University of Limerick, Limerick, Ireland (K Elmusharaf); University of California, Davis, CA, USA (R Engle-Stone); Maternal and Child Survival Program, Washington, DC, USA (K Gilroy, B Rawlins); John Snow Incorporated, Crystal City, VA, USA (K Gilroy); Unitaid, Geneva, Switzerland (T Guenther); Association for Young People's Health, London, UK (A Hagell); Feinberg School of Medicine, Northwestern University, Evanston, IL, USA (L R Hirschhorn); University of Alberta, Edmonton, AB, Canada (S Hodgins); University of Yamanashi, Kofu, Japan (S Horiuchi); Independent Consultant, Princeton, IL, USA (L Kann); Heidelberg Institute of Global Health, Heidelberg, Germany (J-L Koulidiati); Harvard T H Chan School of Public Health (M E Kruk, H H Leslie, K E A Semrau); Post Graduate Institute of Medical Education and Research, Chandigarh, India (R Kumar); London School of Hygiene \& Tropical Medicine, London, UK (J Lawn, T Marchant, J Schellenberg); WHO Collaborating Centre for Maternal and Child Health, Institute for Maternal and Child Health IRCCS Burlo Garofolo, Trieste, Italy (M Lazzerini); South African Medical Research Council, Tygerberg, South Africa (C Mathews); Bill \& Melinda Gates Foundation, Seattle, WA, USA (C-H Mershon, E Piwoz, R Rawat, K Somers, S Subramanian); United Nations Population Fund, New York, NY, USA (J P Monet); Independent Consultant, Stockholm, Sweden (H Newby); United States Agency for International Development, Washington, DC, USA (P Ram); Department of Health Services Management, Faculty of Health Management and Medical Informatics, Tabriz University of Medical Sciences, Tabriz, Iran (V Sadeghi); Ariadne Labs, Boston, MA, USA (K E A Semrau); Brigham \& Women's Hospital, Boston, MA, USA 
(K E A Semrau); Centers for Disease Control and Prevention, Atlanta, GA, USA (F Serbanescu); Fistula Care Plus Project, EngenderHealth, Washington, DC, USA (V Tripathi); Save the Children, Washington, DC, USA (L Vaz, S Wall); and Witten/Herdecke University, Witten, Germany (R Weigel).

\section{Declaration of interests}

We declare no competing interests.

\section{Acknowledgments}

The Effective Coverage Think Tank series was funded by a grant from the UK Department for International Development. The authors alone are responsible for the views expressed in this Health Policy paper, which do not necessarily represent the views, decisions, or policies of the institutions with which the authors are affiliated.

\section{References}

1 UN. Transforming our world: the 2030 Agenda for Sustainable Development. United Nations, 2015. https:// sustainabledevelopment.un.org/post2015/transformingourworld (accessed March 11, 2020).

2 Shengelia B, Tandon A, Adams OB, Murray CJ. Access, utilization, quality, and effective coverage: an integrated conceptual framework and measurement strategy. Soc Sci Med 2005; 61: 97-109.

3 Jannati A, Sadeghi V, Imani A, Saadati M. Effective coverage as a new approach to health system performance assessment: a scoping review. BMC Health Serv Res 2018; 18: 886.

4 Amouzou A, Leslie HH, Ram M, et al. Advances in the measurement of coverage for RMNCH and nutrition: from contact to effective coverage. BMJ Glob Health 2019; 4 (suppl 4): e001297.

5 Nguhiu PK, Barasa EW, Chuma J. Determining the effective coverage of maternal and child health services in Kenya, using demographic and health survey data sets: tracking progress towards universal health coverage. Trop Med Int Health 2017; 22: 442-53.

6 Leslie HH, Malata A, Ndiaye Y, Kruk ME. Effective coverage of primary care services in eight high-mortality countries. BMJ Glob Health 2017; 2: e000424.

7 Colson KE, Zúñiga-Brenes P, Ríos-Zertuche D, et al. Comparative estimates of crude and effective coverage of measles immunization in low-resource settings: findings from Salud Mesoamérica 2015. PLoS One 2015; 10: e0130697.

8 Lozano R, Soliz P, Gakidou E, et al. Benchmarking of performance of Mexican states with effective coverage. Lancet 2006; 368: 1729-41.

9 Greenberg AE, Hader SL, Masur H, Young AT, Skillicorn J, Dieffenbach CW. Fighting HIV/AIDS in Washington, D.C. Health Aff (Millwood) 2009; 28: 1677-87.

10 Tanahashi T. Health service coverage and its evaluation. Bull World Health Organ 1978; 56: 295-303.

11 WHO. WHO recommendations on maternal health: guidelines approved by the WHO Guidelines Review Committee. Geneva: World Health Organization, 2017.

12 WHO. WHO recommendations on newborn health: guidelines approved by the WHO Guidelines Review Committee. Geneva: World Health Organization, 2017.

13 WHO. WHO recommendations on child health: guidelines approved by the WHO Guidelines Review Committee. Geneva: World Health Organization, 2017.

14 WHO. WHO recommendations on adolescent health: guidelines approved by the WHO Guidelines Review Committee. Geneva: World Health Organization, 2017.

15 WHO. WHO recommendation on respectful maternity care during labour and childbirth. The WHO Reproductive Health Library, 2018. https://extranet.who.int/rhl/topics/preconception-pregnancychildbirth-and-postpartum-care/care-during-childbirth/whorecommendation-respectful-maternity-care-during-labour-andchildbirth (accessed March 11, 2020)
16 Heywood A, Rohde J. Using information for action: a manual for health workers at facility level. Pretoria; The EQUITY Project, 2002.

17 Ng M, Fullman N, Dieleman JL, Flaxman AD, Murray CJ, Lim SS. Effective coverage: a metric for monitoring Universal Health Coverage. PLoS Med 2014; 11: e1001730.

18 Kruk ME, Gage AD, Arsenault C, et al. High-quality health system in the Sustainable Development Goals era: time for a revolution. Lancet Glob Health 2018; 6: e1196-252.

19 Every Woman Every Child. Global strategy for women's, children's and adolescents' health (2016-2030). Geneva: World Health Organization, 2015. https://www.who.int/life-course/partners/ global-strategy/globalstrategyreport2016-2030-lowres.pdf (accessed March 26, 2020).

20 Boschi-Pinto C, Labadie G, Dilip TR, et al. Global implementation survey of Integrated Management of Childhood Illness (IMCI): 20 years on. BMJ Open 2018; 8: e019079.

21 Patel S, Pfaffmann Zambruni J, Palazuelos D, et al. Rethinking the scale up of Integrated Management of Childhood Illness. BMJ 2018; 362: k2993.

22 UN Statistical Commission. SDG indicator 3.8.1 metadata 2018. Geneva: World Health Organization, 2018. https://unstats.un.org/ sdgs/metadata/files/Metadata-03-08-01.pdf (accessed March 11, 2020).

23 Hogan DR, Stevens GA, Hosseinpoor AR, Boerma T. Monitoring universal health coverage within the Sustainable Development Goals: development and baseline data for an index of essential health services. Lancet Glob Health 2018; 6: e152-68.

24 Penchansky R, Thomas JW. The concept of access: definition and relationship to consumer satisfaction. Med Care 1981; 19: 127-40.

25 Kroeger A. Anthropological and socio-medical health care research in developing countries. Soc Sci Med 1983; 17: 147-61.

26 Ozawa S, Yemeke TT, Evans DR, Pallas SE, Wallace AS, Lee BY. Defining hard-to-reach populations for vaccination. Vaccine 2019; 37: 5525-34.

27 Roder-DeWan S, Gage AD, Hirschhorn LR, et al. Expectations of healthcare quality: a cross-sectional study of internet users in 12 low- and middle-income countries. PLoS Med 2019; 16: e1002879.

28 WHO. WHO guideline: recommendations on digital interventions for health system strengthening. Geneva: World Health Organization, 2019

29 Maina I, Wanjala P, Soti D, Kipruto H, Droti B, Boerma T. Using health-facility data to assess subnational coverage of materna and child health indicators, Kenya. Bull World Health Organ 2017; 95: 683-94.

30 WHO. Consolidated guidelines on person-centred HIV patient monitoring and case surveillance. Geneva: World Health Organization, 2017.

31 Willey B, Waiswa P, Kajjo D, et al. Linking data sources for measurement of effective coverage in maternal and newborn health what do we learn from individual- vs ecological-linking methods? J Glob Health 2018; 8: 010601

(C) 2020. This is an Open Access article published under the CC BY-NC-ND 3.0 IGO license which permits unrestricted use, distribution, and reproduction in any medium, provided the original work is properly cited. In any use of this article, there should be no suggestion that WHO endorses any specific organisation, products or services. The use of the WHO logo is not permitted. This notice should be preserved along with the article's original URL. 